\title{
People with enduring mental health problems described the importance of communication, continuity of care, and stigma
}

Kai J. Crosland A. Perspectives of people with enduring mental ill health from a community-based qualitative study. Br Gen Pract 2001 Sep;51:730-6.

\section{QUESTION: How do people with enduring mental health problems experience health care?}

Design

Community based qualitative study.

\section{Setting}

4 general practices serving a disadvantaged locality of 5 wards in $\{\text { Newcastle upon Tyne }\}^{*}, \mathrm{UK}$.

\section{Participants}

32 people ( $56 \%$ men, mean duration of mental illness of 21 y) who were identified by their general practitioner as disabled by enduring mental ill health $(\geqslant 2$ y impaired social behaviour and inability to keep a job, maintain self care, complete domestic chores, or participate in recreational activities). Exclusion criteria were age $<16$ years, dementia or other organic brain disorder, or a learning disability.

\section{Methods}

Individual interviews of 45 minutes to 2 hours were audiotaped and transcribed verbatim. Theoretical saturation occurred after 34 interviews; 2 interviews were excluded because the respondents did not acknowledge any experience of mental illness. Data were analysed using grounded theory methods. Emerging findings were tested in 21 group interviews with local health professionals.

\section{Main findings}

Good therapeutic relationships were regarded as central in participants' contacts with primary care and mental health services. Several related themes emerged. Communication in building therapeutic relationships: in good therapeutic relationships, professionals had effective listening skills and showed empathy and understanding, which allowed participants to express their concerns, negotiate options, and build trust. Continuity of therapeutic relationships: participants highlighted the importance of building a continuing relationship with one person over time. Frequent changes in healthcare providers and repeatedly reviewing medical histories were frustrating for participants. Wrestling with stigma: influence of the healthcare setting: participants worried about where their healthcare contacts occurred, and the potential that they might be stigmatised by association with other patients and mental health settings. Conversely, another theme highlighted the value of mutual patient support and specialist settings: participants who had received prolonged inpatient treatment valued the shared contact with and support of other patients. As they recognised their own illness, they accepted other patients, became less concerned about where they were seen, and focused on developing therapeutic relationships with mental health professionals. Experience of social exclusion: participants described a "constant juggling between the competing demands of their mental ill health, trying to minimise the effects of stigma, and coping with poverty, fear and exclusion." Taking control of their lives was hampered by common experiences of victimisation, crime, social isolation, and lack of access to material resources. These constraints increased stress, curtailed opportunities to promote wellbeing, and reduced coping capacity. Potential contribution of professional care: most participants considered the value of positive therapeutic relationships within the context of social exclusion and stigma. Health professionals were an important source of support over time that helped them to overcome barriers, identify solutions, and establish control over their lives.

\section{Conclusion}

People with enduring mental health problems valued empathic and understanding professionals with effective listening skills, and a continuing relationship with one professional. They feared social stigma and experienced social exclusion related to their mental illness.

*Information provided by author.

\author{
Sources of funding: \\ Newcastle West Primary \\ Care Group and \\ Newcastle City Health \\ Trust. \\ For correspondence: \\ Dr J Kai, Department of \\ Primary Care and \\ General Practice, \\ University of \\ Birmingham, Edgbaston, \\ Birmingham, UK. \\ j.p.kai@bham.ac.uk \\ A modified version of this \\ abstract appears in \\ Evidence-Based Mental \\ Health.
}

\section{COMMENTARY}

The study by Kai and Crosland examined the healthcare experiences of people with mental health problems. The authors used sound methods for grounded theory, although it was puzzling that they chose to validate the themes with groups of providers rather than patients. The study is unique as its participants were identified from a primary care setting rather than from a specialised psychiatric setting.

Psychiatric nurses have long claimed that the therapeutic relationship is the essence of practice. ${ }^{1}$ However, recently this focus has been questioned, and a shift to a more biological focus for psychiatric nursing has been proposed. ${ }^{2}{ }^{3}$ The study findings support the belief that the therapeutic relationship is the backbone upon which all other care is delivered. Interestingly, no themes related to a more biological perspective emerged. Participants were disturbed by breaks in the continuity of care. The reported problems resulting from inconsistent care providers are similar to those of an earlier study, which found that even brief changes in providers for vacation coverage could create difficulties. ${ }^{4}$ Practice implications arise from an understanding that continuity of care is not simply continuity of a plan of care, but also involves continuity of the care provider.

The study participants placed the therapeutic relationship within the broader context of social struggles such as stigma and poverty. Such perspectives challenge us to consider problems at broad community levels as well as interpersonal levels. Interestingly, participants sometimes felt that providers were also part of the problem. In particular, they sometimes felt stigmatised by the care providers themselves. Practitioners reading this paper may find it useful to reflect upon the ways they may communicate acceptance or rejection of common stereotypes and stigma.

Cheryl Forchuk, RN, PhD Associate Professor/Nurse Scientist University of Western Ontario/Lawson Health Services Research London, Ontario, Canada

1 Peplau HE. Interpersonal relations in nursing: a conceptual frame of reference for psychodynamic nursing. New York: GP Putnam and Sons, 1952

2 Gournay K. Schizophrenia: a review of the contemporary literature and implications for mental health nursing theory, practice and education. I Psychiatr Ment Health Nurs 1996;3:7-12.

3 McCrone SH. The impact of the evolution of biological psychiatry on psychiatric nursing. J Psychosoc Nurs Ment Health Serv 1996;34:38-46.

4 Forchuk C. The orientation phase of the nurse-client relationship: how long does it take? Perspectives in Psychiatric Care 1992;28:7-10. 